NOVEL PLATINUM AGENTS AND MESENCHYMAL STROMAL CELLS FOR THORACIC

\title{
MALIGNANCIES: STATE OF THE ART AND FUTURE PERSPECTIVES
}

Francesco Petrella ${ }^{1,2^{*}}$, Isabella Rimoldi ${ }^{3}$, Giorgio Facchetti ${ }^{3}$ and Lorenzo Spaggiari ${ }^{1,2}$

${ }^{1}$ Department of Thoracic Surgery, European Institute of Oncology, Milan, Italy

${ }^{2}$ Department of Oncology and Hemato-oncology, University of Milan, Italy

${ }^{3}$ Department of Pharmaceutical Sciences, University of Milan, Italy

${ }^{*}$ Corresponding Author:

Francesco Petrella, MD

Department of Thoracic Surgery

European Institute of Oncology - Via Ripamonti, 435

Department of Oncology and Hemato-oncology, University of Milan, Milan, Italy 20141 Milan - ITALY

Tel: 0039.0257489362

Fax: 0039.0294379218

E mail: francesco.petrella@ieo.it

francesco.petrella@unimi.it

Word count: abstract 193; text: 4287 


\section{ABSTRACT}

\section{Introduction}

Non-small cell lung cancer and malignant pleural mesothelioma represent two of the most intriguing and scrutinized thoracic malignancies, presenting interesting perspective of experimental development and clinical applications.

\section{Areas covered}

In non-small cell lung cancer advanced-stage disease, molecular targeted therapy is the standard first-line treatment for patients with identified driver mutations; on the other hand, chemotherapy is the standard treatment for patients without EGFR mutations or ALK rearrangement or those with unknown mutation status. Once considered an ineffective therapy in pulmonary neoplasms, immunotherapy has been now established as one of the most promising therapeutic options.

Mesenchymal stromal cells are able to migrate specifically towards solid neoplasms and their metastatic localizations when injected intravenously. This peculiar cancer tropism has opened up an emerging field to use them as vectors to deliver antineoplastic drugs for targeted therapies.

\section{Expert opinion}

Molecular targeted therapy and immunotherapy are the new alternatives to standard chemotherapy. 
Mesenchymal stromal cells are a new promising tool in oncology and - although not yet utilized in the clinical practice, we think they will represent another main tool for cancer therapy and will probably play a leading role in the field of nanovectors and molecular medicine.

KEYWORDS: non-small cell lung cancer; malignant pleural mesothelioma; immunotherapy; targeted therapy; mesenchymal stromal cell; drug loading and delivery. 


\section{INTRODUCTION}

Lung cancer is one of the most frequently diagnosed cancer and is the leading cause of cancer-related death worldwide. ${ }^{1}$ Non-small-cell lung cancer (NSCLC) - including squamous cell carcinoma, adenocarcinoma and large-cell carcinoma subtypes accounts for approximately $85 \%$ of all new lung neoplasms while small-cell lung cancer $(\mathrm{SCLC})$ represents the remaining $15 \%{ }^{2}$

Overall primary pulmonary tumors represent $13 \%$ of the total newly diagnosed cancer cases and $19.4 \%$ of total cancer related deaths, worldwide every year. ${ }^{1}$

Cigarettes smoking is the main risk factor for developing lung cancer: in fact, smoke exposure can lead to a well-described series of morphological modifications of the bronchial epithelium structure, evolving from basal cell hyperplasia to metaplasia, severe dysplasia to carcinoma in situ and, finally, frank carcinoma. ${ }^{3}$

These pathologic modifications are mainly associated with the squamous subtype; on the contrary, although primary lung adenocarcinoma may develop in case of high carcinogen exposure and underlying pulmonary damage, it is commonly described as the more frequent histologic subtype in never-smoker patients with low carcinogen exposure; its progression is related to less well-characterized pre-neoplastic lesion called atypical adenomatous hyperplasia. SCLC also commonly occurs in case of high 
carcinogen exposure, but it derives from pulmonary neuroendocrine cells and does not have well-characterized pre-malignant lesions. ${ }^{1}$

From the therapeutic point of view, although NSCLC is a heterogeneous disease and its treatment should be personalized according to the patient's characteristics, it is generally classified into three treatment categories: standard curative treatment for patients with resectable NSCLC is surgical resections; multimodal therapy (chemotherapy, surgery and radiotherapy) is indicated for locally advanced non metastatic patients; metastatic patients can be treated by standard chemotherapy, molecular targeted therapy or immunotherapy..$^{5-7}$

Malignant pleural mesothelioma (MPM) is an uncommon fatal asbestos-related tumor originating in the mesothelial cells of the pleurae. It can occur at any place where mesothelial cells de-differentiate from mesenchymal cells, including the pericardium, peritoneum, tunica vaginalis of the ovary and testis, although both pleurae (visceral and parietal pleura) are the most common affected structures. ${ }^{8,9}$ The incidence of MPM in in Japan and Europe are projected to peak in 2025 and 2020 respectively, while the incidence in the USA has remained stable at 3000 deaths per year since peaking in $2004 .^{10}$

Surgical resection is the best therapeutic approach in early stages MPM (I, II and selected NO stage III) without distant diffusion as well as radiotherapy as complementary or symptomatic treatment. ${ }^{11} \mathrm{~A}$ platinum-based doublet with a third- 
generation antifolate (pemetrexed (PMX) or raltitrexed) is the present front-line standard of care; on the contrary, there are no approved second-line treatments for MPM which remains a disease setting to test the efficacy of new therapeutic agents. ${ }^{8}$ Both NSCLC and MPM - although different clinical and epidemiologic entities nowadays represent two of the most intriguing and scrutinized thoracic malignancies, presenting interesting perspective of experimental development and daily clinical applications; the aim of the present review is to focus on preclinical and clinical aspects of medical therapy of NSCLC and MPM. 
In NSCLC advanced-stage disease, molecular targeted therapy is the standard firstline treatment for patients with identified driver mutations; on the other hand, systemic cytotoxic chemotherapy is the standard treatment for patients without EGFR mutations or ALK rearrangement or those with unknown mutation status.

\section{NSCLC chemotherapy}

Standard first-line chemotherapy should be one of the platinum-based doublet chemotherapy regimens for patients with unknown mutational status or those without driver mutations. ${ }^{12,13}$

It has been demonstrated that pemetrexed-based combinations are superior to gemcitabine-based combinations for patients suffering from adenocarcinoma while efficacy is similar between the standard regimens (platinum plus any of paclitaxel, gemcitabine, docetaxel and vinorelbine). ${ }^{1}$

When bevacizumab - that is a VEGF-specific monoclonal antibody - is added to standard chemotherapeutic regimen, it improves efficacy; on the contrary, it is contraindicated in patients affected by squamous cell carcinoma or presenting haemoptysis. ${ }^{14}$ 
In patients with non-squamous histology, if first-line treatment evokes a response or stabilizes disease, maintenance treatment with single-agent pemetrexed can be taken into consideration. ${ }^{15}$

With the aim to research new drugs especially for intervention of drug-resistance lung cancer, different hybrid molecules between an oleanolic acid derivative (CDDO) and a diazeniumdiolate derivative (Figure $\mathbf{1}$, series I) was synthetized and in vitro evaluated on drug resistant A549/Taxol cells. One compound of the series revealed an anti-proliferative activity due to synergic effects of two drugs with enhanced generation of high levels of NO/ROS and increased the inhibition of stress response pathway through effect on Lon protease. ${ }^{16}$ A new series of 2-oxo-3,4diihydropyrimidinyl derivatives (Figure 1, series II) was synthetized and evaluated as suppressor of the proliferation of H520 NSCLC through irreversible binding with the FGFR1-3 target kinase and revealed a high potency also against FGFR4, resulting inhibitors of the FGFR family. ${ }^{17}$ Valid alternative anticancer alkaloids resulted the marine-derived molecules capable to interact with different targets. A series of analogues of renieramycin $M$ (Figure 1, series III) possessed a nanomolar concentration cytotoxic activity against $\mathrm{H} 292$ and H490 human NSCLC cell lines. Investigation about the mechanism of action is in progress, allowing a future clinical application of the molecules considering the high activity demonstrated on lung cancer. $^{18}$ 


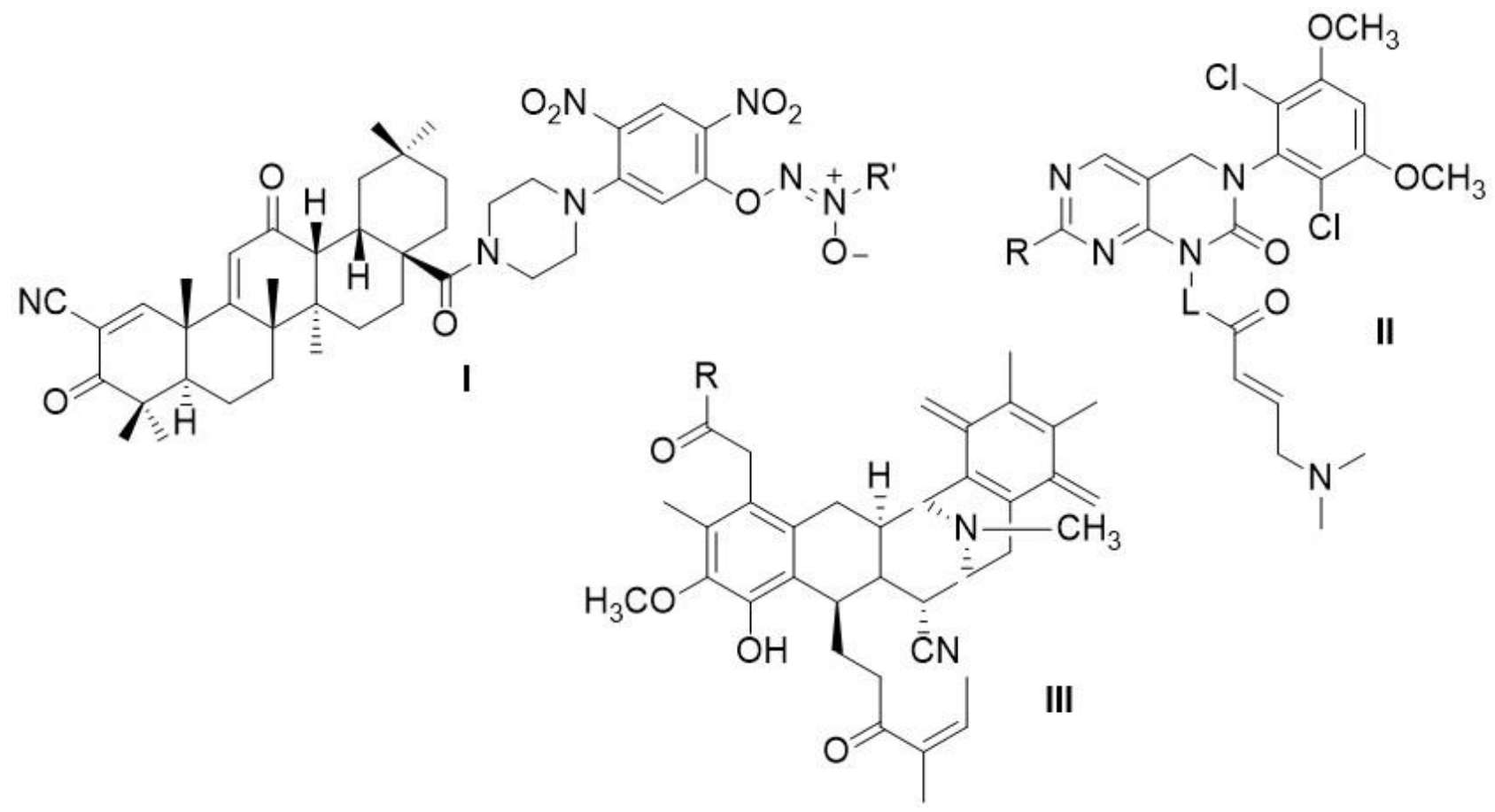

Figure 1. Example of new compounds under evaluation for the treatment of NSCLC. 


\section{NSCLC new platinum drugs in vitro evaluation}

Platinum-based chemotherapy has been the standard first-line treatment for unselected patients with metastatic NSCLC, with median survivals of 8-12 months even if the therapeutic efficiency changes remarkably among patients. The main target of platinum drugs is DNA forming platinum-DNA adducts through intra- and interstrand crosslink. The resistance to these drugs largely depends on the enhanced DNA repair systems and the generic variations of DNA repair genes were important for the resistance and for the possibility to use their polymorphisms as platinum response genetic markers. ${ }^{19}$ The possibility to bypass the resistance in NSCLC patients is the base of the synthesis of new platinum(II) and platinum(IV) complexes, in vitro evaluated as promising anticancer agents.

In the case of platinum(II) complexes, a novel class of homo- and heteroleptic complexes of ONN-donor hydrazone and 4-picoline (Figure 2, series IV) showed a potent cytotoxic effect comparable to cisplatin on lung cancer cell lines H460, suppressing cell growth effectively triggering apoptosis and inducing changes in morphology of cancer cells inhibiting also cell migration in lung cancer. ${ }^{20} \mathrm{~A}$ series of trans-platinum(II) oxadiazole complexes with 7-nitro-1,3,5-triaza-adamantane or hexamethylenetetramine ligands (Figure 2, series V) was evaluated in vitro as cytotoxic agents on lung cancer cell lines A549 revealing that six of mononuclear complexes of the series were more active in A546 than cisplatin at $2.5 \mu \mathrm{M}$ concentration. ${ }^{21}$ A strategy for overcoming the drawbacks due to the use of 
platinum(II) complexes consists in the oxidation of active square-planar platinum(II) species to an octahedral platinum(IV) complex that can act as prodrug through the activation by reduction process occurred in biological environment. Among a series of platinum(IV) complexes conjugated with phenstatin analogue as inhibitors of microtubule polymerization, the complex VI (Figure 2) revealed to cause apoptotic cell death in human NSCLC cell line $\mathrm{NCl}-\mathrm{H} 460$ both in vitro and xenograft mouse model in vivo through the mitochondrial mediated pathway and induced cell-cycle arrest at the G2/M phase. ${ }^{22}$ Another interesting series of platinum(IV) complexes, the cyclometallated iodido complex series VII (Figure 2), showed a cytotoxic activity against human lung cancer cell lines A-549 resulting topoisomerase I inhibitors. In particular two compounds of the series suppressed A-459 growth by apoptotic induction and call cycle arrest, and induced high levels of reactive oxygen species (ROS) involved in mitochondria permeabilization, consequently causing the realese of cytochrome $\mathrm{c}$ and finally the apoptosis. ${ }^{23}$ 


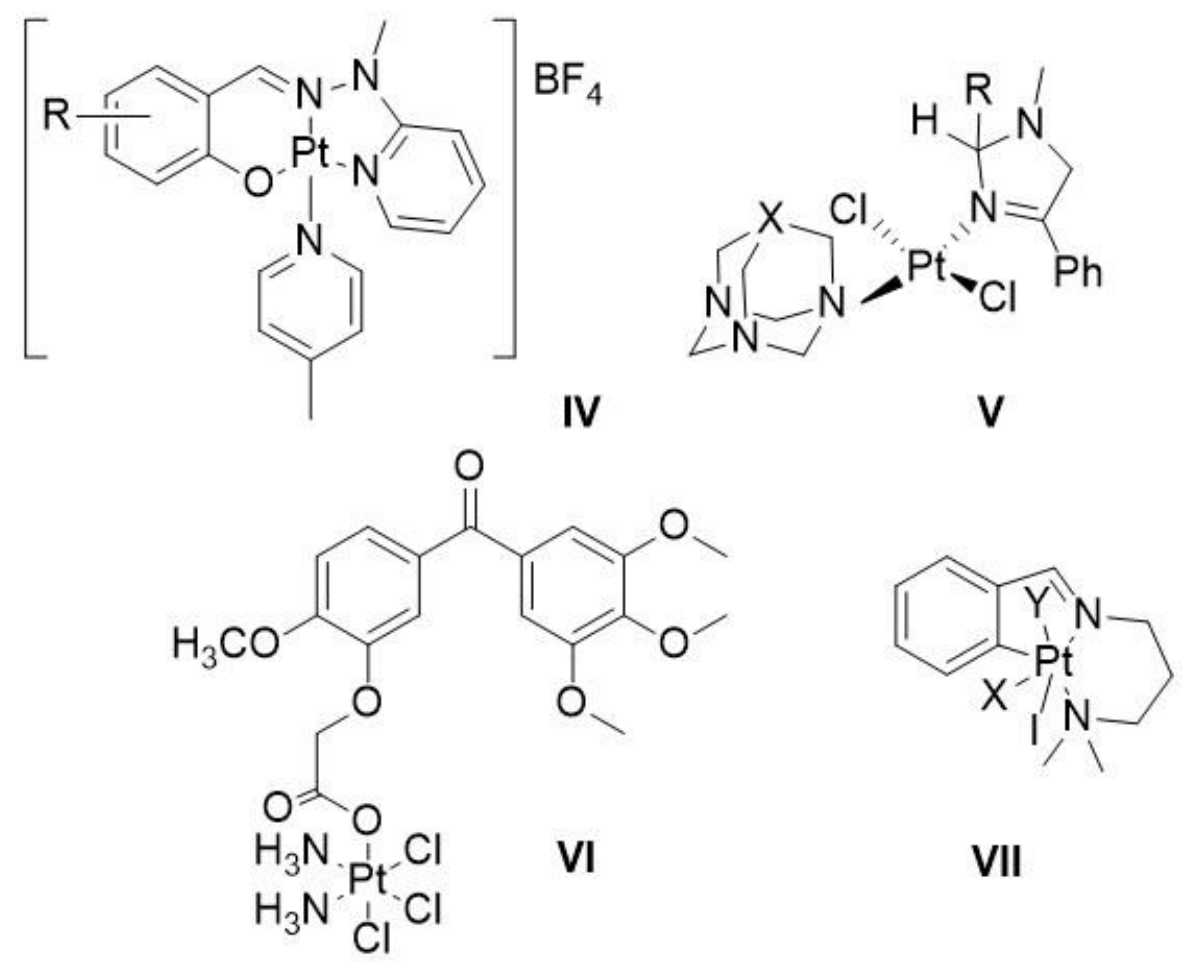

Figure 2. Some examples of novel platinum(II) and platinum(IV) complexes evaluated on NSCLC. 


\section{NSCLC molecular targeted therapy}

A wide variety of lung cancers with different characteristics exists, being the identification of driver oncogene mutations of paramount importance to understand these differences. These acquired genetic mutations in kinases result in constitutive signaling and, in susceptible cells, this leads to oncogenic transformation that is almost independent from other alterations. Oncogenes involved in NSCLC are activating mutations in the epidermal growth factor receptor (EGFR) gene and translocations of the anaplastic lymphoma kinase (ALK) gene. The EGFR and ALK driver mutations are mainly observed in occurred adenocarcinomas (10-60\%), with a strong influence of smoking habits, being, in fact, more frequent in never smokers. ${ }^{24}$ Several targeted therapies have been successfully applied for treating tumors harboring EGFR and ALK mutations, presenting satisfactory response rates; however, it is well known that neoplasms eventually acquire resistance to these targeted therapies, although the mechanisms by which resistance occurs are not yet fully understood.

Less frequent mutations have also been described as targets, like translocations of RET, ROS1 and receptor tyrosine kinases, mutations in BRAF, MET, HER2 and amplifications of MET, HER2 and fibroblast growth factor receptor 1. Although mutations in the tumor suppressor genes TP53 and RB1 are commonly reported in all lung cancer histotypes, they are not yet therapeutically applicable. 
In patients with advanced stage NSCLC, first-line EGFR TKIs are superior to standard platinum-based chemotherapy. ${ }^{25}$ The vast majority of patients would respond to EGFR TKIs but they will then almost always develop resistance, although the pattern of resistance is variable. In patients presenting systemic progressive disease with symptoms or fast progression, EGFR TKI therapy should be discontinued, and therapeutic protocol shifted to standard platinum-based chemotherapy. The most common cause of resistance is the T790M mutation, representing about $60 \%$ of all cases of EGFR TKI resistance. ${ }^{26}$

Similar to mutations in EGFR, rearrangements in ALK are driver oncogenes, and patients with ALK-positive lung cancer are highly responsive to TKIs. ${ }^{27}$

Crizotinib was the first TKI approved for first and subsequent lines of treatment of ALK-positive NSCLC, showing superior response rates and prolonged progression-free survival when compared to doublet chemotherapy; thus, all patients with adenocarcinoma should be tested for ALK rearrangement and first-line crizotinib should be offered to patients with ALK-positive lung cancer.

ROS1 rearrangement is another driver oncogene that is highly responsive to crizotinib. ${ }^{28}$ An activating mutation in the ROS1 gene is observed in about $1 \%$ of lung adenocarcinomas, occurring most frequently in non-smoker young patients. The presence of these activating mutations is the therapeutic target for the lowmolecular-weight selective inhibitor of the ALK and ROS1 tyrosine kinase receptors - 
crizotinib. $^{29}$

BRAF is a member of the serine/threonine kinase RAF family that is regulated by binding to RAS and directly activating MEK1/2, which can further phosphorylate ERK1/2. BRAF mutations are detected in approximately $2 \%$ to $4 \%$ of lung cancer, mainly adenocarcinomas. In vitro preclinical models of NSCLC demonstrated that both vemurafenib and trametinib were effective as single agents in BRAF V600E mutant cells; Sorafenib has also been reported to be active in patients with BRAF mutant NSCLC. ${ }^{30}$

Despite an initial fast response to crizotinib, many patients develop resistance to this drug: one mechanism causing this resistance is a secondary mutation within the kinase domain of EML4-ALK, similar to that of T790M conferring resistance to EGFRTKIs in tumors with activating mutations of EGFR. Moreover, amplification of the ALK fusion gene and up-regulation of bypass signaling pathways mediated by EGFR, human epidermal growth factor receptor 2 (HER2), c-KIT, or the insulin-like growth factor-1 receptor have been found as mechanisms of crizotinib resistance. ${ }^{31}$

Alectinib as well as ceritinib is highly selective second-generation ALK-TKIs developed for patients with NSCLC positive for ALK rearrangement; in particular Alectinib was found to possess potent antineoplastic activity against $A L K$ fusion-positive NSCLC cells harboring the most common crizotinib resistance mutations. ${ }^{31}$ 


\section{NSCLC immunotherapy}

Tumors develop genetic modifications to protect themselves from an effective human immune response. Immunotherapeutic strategies for the treatment of lung cancer involve a complex interaction between various components of the innate and adaptive immune systems. The involved cell types include CD8+ T lymphocytes (cytotoxicT-cells), Th1 and Th2 subtypes of CD4+ T lymphocytes (helper T-cells), NK, 
Treg and macrophages. Each cell type plays a specific role in the immune cascade that ultimately leads to a cytotoxic response against the tumor cells. ${ }^{32}$

The vast majority of solid cancers avoid host immune response and elimination by subversion of normal regulatory signals, like cytotoxic $\mathrm{T}$ lymphocyte-associated antigen 4 (CTLA4) - involved in T cell priming - and the programmed cell death protein 1 (PD1) and PD1 ligand 1 (PDL1), involved in T cell killing. ${ }^{33}$

Many NSCLCs, in fact, disclose upregulated expression of PDL1, which binds to PD1 thus inactivating PD1-expressing T lymphocytes. A group of patients show effective and prolonged responses when treated with antibodies active on this pathway. ${ }^{34}$

Once considered an ineffective therapy in pulmonary neoplasms, immunotherapy has been now established as one of the most promising therapeutic options: nivolumab, an anti-programmed death 1 (PD-1) antibody, and ipilimumab, an anti-cytotoxic Tlymphocyte antigen 4 (CTLA-4) antibody, are immune checkpoint inhibitors with complementary mechanisms of action. ${ }^{35}$

Patients with advanced NSCLC - without targetable mutations - can be treated using single agent immunotherapy as first-line therapy, depending on PD-L1 status. If the PD-L1 expression is $\geq 50 \%$, then pembrolizumab can be considered for first-line therapy. Stage IV NSCLC patients who are intolerant to platinum-based chemotherapy or progress on or after platinum-based therapy, can be treated by pembrolizumab if PD-L1 tumor expression is $\geq 1 \%$. Atezolizumab and Nivolumab have been approved for 
second-line treatment for stage IV NSCLC irrespective of PD-L1 tumor expression and so they can be considered to treat patients without any detectable expression of PDL1. Durvalumab can be used as consolidation therapy for up to 12 months in patients with unresectable stage III NSCLC, presenting good response to chemoradiation. ${ }^{32}$

\section{MPM chemotherapy}

Currently approved first-line treatment for MPM is chemotherapy with pemetrexed plus cisplatin, which is associated with a median overall survival of approximately 12 months. ${ }^{36,37}$

At present, there is no approved second-line treatments: therapeutic alternatives after ineffective response of first-line treatment are pemetrexed alone - in case of pemetrexed-naive in first line - platinum chemotherapy rechallenge (if patients had response in first line), vinorelbine or gemcitabine monotherapy, or participation in a clinical trial. ${ }^{38}$ Median overall survival with second-line therapy ranges from 5 to 10 months, ${ }^{39}$ thus showing globally quite disappointing results. 
Drug repositioning, using already approved drugs for new indications, is a promising strategy for identifying active molecules in a more rapid and less expensive way. In this regard, pyrvinium pamoate (PP) (Figure 3, compound VIII), a classical FDAapproved anthelmintic, has recently attracted a great interest for its established inhibitory effect on the $\mathrm{Wnt} / \beta$-catenin pathway known for playing a particularly important role in MPM progression. ${ }^{40}$

The data showed that PP was able to decrease $\beta$-catenin levels in a time- and dosedependent manner as well as to reduce the expression of several key genes such as the VEGFA (vascular endothelial growth factor A) and MET (mesenchymal-epithelial transition) factor, whose expression are related to the invasiveness and aggressiveness of the MPM. Furthermore, it was found that PP acted synergistically with well-known approved chemotherapeutics such as doxorubicin, resulting practical and beneficial because of the already proven PP safety.

Starting from a similar approach based on the reprofiling of known active molecules for new applications, a series of derivatives were synthesized from naftodipil, an $\alpha 1$ adrenoceptor blocker, clinically used and applied to the treatment of benign prostate hyperplasia but recently found to be able to induce apoptosis in MPM cells by activating caspase- 8 and the effector caspase-3.

Among the many derivatives that were synthesized and tested on different human MPM cell lines, HUHS1015 (Figure 3, compound IX) resulted the most active of the series in reducing cell viability with a greater potential than both cisplatin and 
paclitaxel at concentrations higher than $30 \mu \mathrm{M}$. Its ability to induce apoptosis of MPM cells, probably through mitochondria, and to interfere with cell-cycle progression make HUHS1015 a promising candidate for the development of a new anticancer drug active on mesothelioma. ${ }^{41}$

Another strategy employed in the effort to design new and more active chemotherapeutics for the treatment of MPM is based on the preparation of hybrid bifunctional agents with the aim to simultaneously inhibit multiple cellular targets involved in MPM growth and progression.

Starting from the established ability to synergistically block cell proliferation between histone deacetylases inhibitors (HDAC) and camptothecin derivatives, a series of new compounds based on the psammaplin A scaffold, known for exerting a potent HDAC inhibitory activity, and camptothecin were synthesized showing a significant antitumor activity, with IC50 values in the nanomolar range on different mesothelioma cell lines (Figure 3, compounds $\mathbf{X}, \mathbf{X I}$ and $\mathbf{X I I )}$ ). Moreover, when tested in vivo on human a mesothelioma model, derivative $\mathbf{X I}$ showed a potent antiproliferative activity along with high tolerability, making it a particularly intriguing compound with encouraging future perspectives. ${ }^{42}$ 


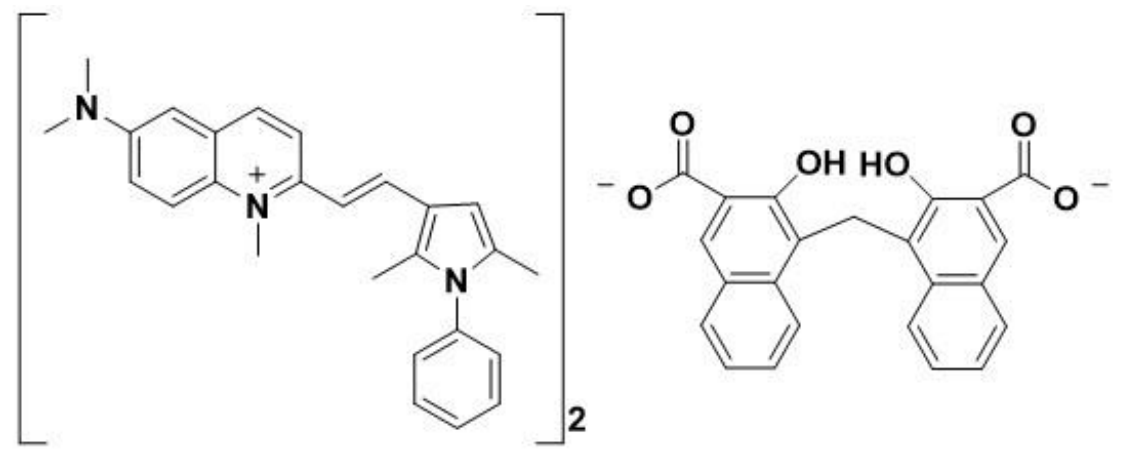

VIII
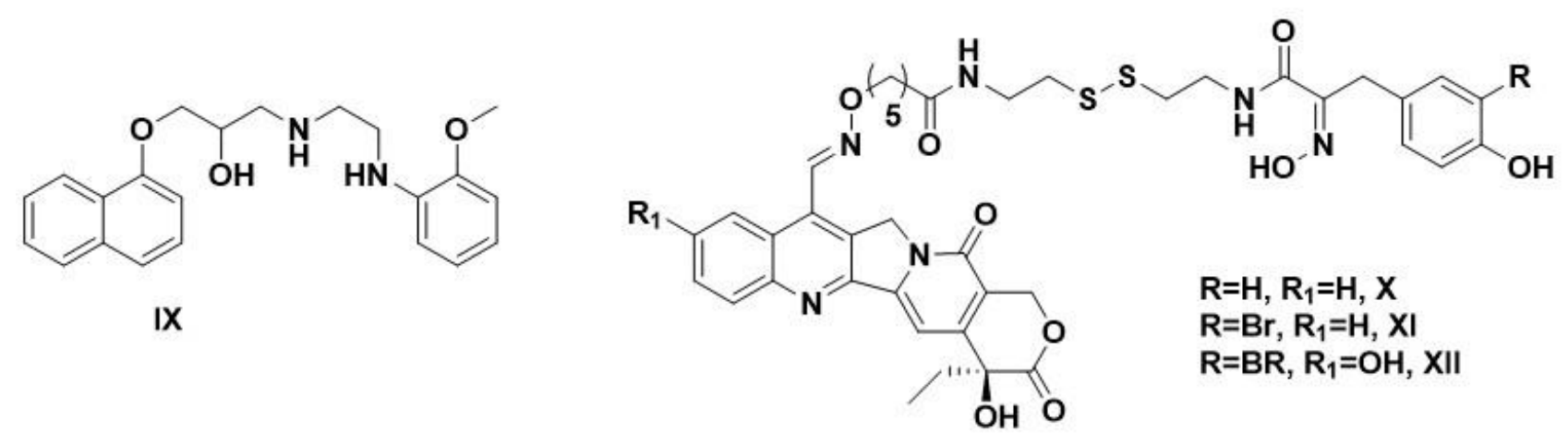

Figure 3. Example of new compounds under evaluation for the treatment of MPM.

\section{MPM new platinum drugs in vitro evaluation}

Currently all chemotherapy protocols provide the use of cisplatin or carboplatin in a dual combinatorial association therapy with the antifolate pemetrexed (or raltitrexed) or with an immunotherapy agent (e.g. bevacizumab). If comorbidities are present carboplatin could be employed due to its milder toxicity profile if compared to cisplatin. Unfortunately, the efficacy of the current platinum-based therapy is really 
modest due to a poor specificity and a higher chemoresistance to these alkylating agents that evoke the need for new compounds alternative to cisplatin to be found. ${ }^{11}$ A first attempt made to overcome cisplatin resistance is based on the high glutathione (GSH) related metabolism that characterized MPM cells. Specifically, the enzyme GSH-S-transferase (GST) not only catalyzes the conjunction of cisplatin with GSH in a detoxification process but it results also involved in those protein-protein interactions with c-Jun $\mathrm{N}$-terminal kinase, a member of the mitogen-activated kinases, whose activity is responsible for triggering cells apoptotic pathways. A dual compound able to combine the cytotoxicity of the metal core with the ability to target GST might represent a novel strategy to overcome MPM chemoresistance to platinum drugs. Dyson and co-workers ${ }^{43}$ developed in this context a Pt(IV) derivative containing a cisplatin equatorial base with two axial molecules of ethacrynic acid (EA), known for being a GST inhibitor. Upon reduction in the hypoxic tumor environment, the so called ethacraplatin (Figure 4, complex XIII) is supposed to release a cisplatin molecule and two equivalents of EA, that should be able to lower cisplatin resistance. As a term of comparison, the cis-diamminobis(ethacrynato)platinum(II) complex with the two EA moieties acting as leaving groups (Figure 4, complex XIII) was also synthesized and tested together with the Pt(IV) analogue on different primary MPM cancer cell lines. ${ }^{44}$ Both complexes resulted more active than carboplatin with the platinum(IV) dual compound more potent in all cases with better resistance factors although less cytotoxic than cisplatin. In a similar manner the axial positions could be occupied by 
different pharmacophores that in principle could offer the possibility to act on different targets in a synergistic way. A recent example provided the mono- and diinsertion of clofibric acid (CA) moieties in axial position of a cisplatin-based platinum(IV) compound (Figure 4, complexes XIV and XV).$^{45}$ The idea is to exploit the ability of CA, normally used for the treatment of dyslipidaemia, to activate the peroxisome proliferator-activated receptor $\alpha$ (PPAR $\alpha)$, that besides controlling the fatty acid metabolism, is also reported to activate apoptosis and to degrade the hypoxia-inducible factor-1 $\alpha$ (HIF-1 $\alpha$ ) known for its implication in tumor growth and progression. Both complexes resulted able to bypass cisplatin resistance with the diclofibric acid derivative more effective on five different MPM cancer cell lines under the hypoxic conditions where cisplatin usually displays a weak cytotoxic activity.

Another series of $\mathrm{Pt}(\mathrm{IV})$ compounds synthesized with the aim of bypassing the antioxidant inactivation exerted by thiol-containing molecules is based on picoplatin, used in a phase II trial as second line therapy for MPM patients with progressive disease only modestly responsive to the first-line treatment (Figure 4, complexes XVI, XVII and XVIII). ${ }^{46}$ In this case, the insertion of picoplatin in an octahedral Pt(IV) complex with carboxylic acids containing differently long carbon chains in axial positions should provide a prodrug easy to be reduced and more stable to GSH mediated detoxification activity, due to its monofunctional mechanism of action. On the tested MPM cancer cell lines, the activity of the picoplatin-based Pt(IV) series of 
complexes showed a cytotoxic activity that increases along with the carbon chain length of the axial substituents. All the Pt(IV) complexes of the series resulted more potent than the parent picoplatin and a lower resistance factors than cisplatin. Among the novel strategies based on platinum compounds for the treatment of MPM malignancies, cationic platinum(II) complexes represent a class of compounds that, by violating the apparently demanded neutrality, could offer a distinct mechanism of action and a different antitumor profile thus allowing to furnish candidate drugs able to overcome cellular resistance and to lower toxicity of cisplatin in MPM patients. Once inside the cell, their cationic nature in fact might allow them to interact more rapidly with the anionic DNA. A series of imidazole based cationic Pt(II) complexes of general formula $\left[\mathrm{Pt}\left(\mathrm{N}-\mathrm{N}^{\prime}\right) \mathrm{N}^{\prime} \mathrm{Cl}\right] \mathrm{X}^{-}$where $N-N^{\prime}$ is an aminomethylimidazole ligand and the $N^{\prime}$ an imidazole ring, both bearing the same alkyl group at the $N 1$ position are very recently synthesized as shown in Figure 4 displaying interesting cytotoxic activity in many cisplatin resistant tumors with the one bearing a hexyl carbon chain at the $N 1$ (complex XXII) resulted more cytotoxic than cisplatin in $\mathrm{NCl}-\mathrm{H} 28$ cancer cell line. ${ }^{47}$ 

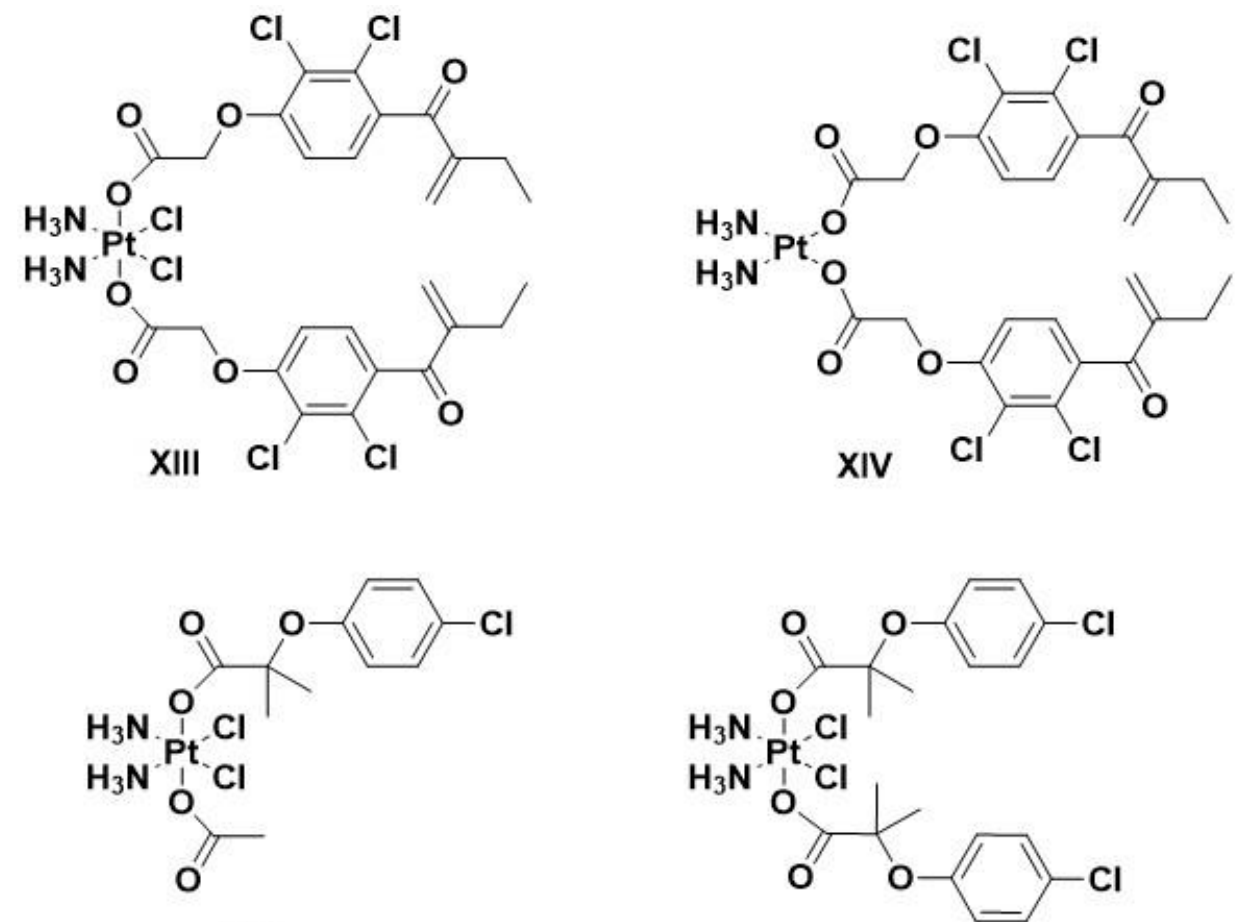

XV
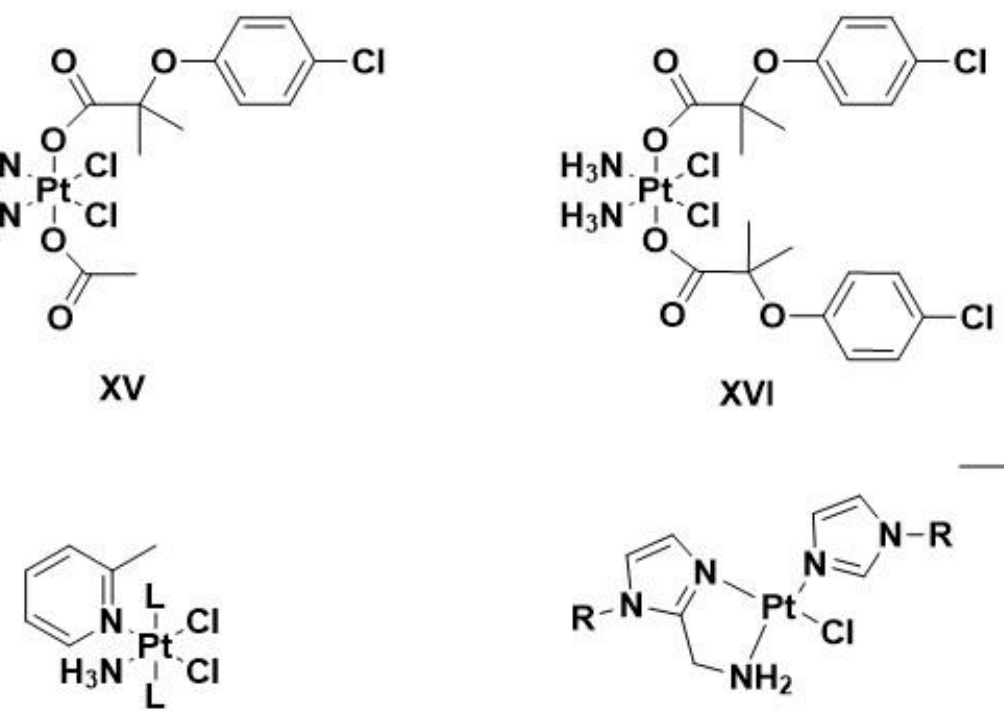

$\mathrm{L}=\mathrm{CH}_{3} \mathrm{COO}-, \mathrm{XVII}$

$\mathrm{L}=\mathrm{CH}_{3} \mathrm{CH}_{2} \mathrm{COO}-, \mathrm{XVIII}$

$\mathrm{L}=\mathrm{CH}_{3} \mathrm{CH}_{2} \mathrm{CH}_{2} \mathrm{COO}-, \mathrm{XIX}$

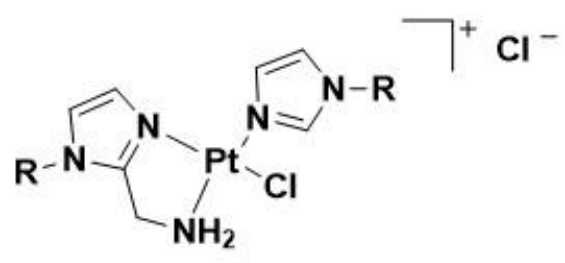

$$
\begin{aligned}
& \mathrm{R}=-\mathrm{CH}_{3}, \mathbf{X X} \\
& \mathrm{R}=-\left(\mathrm{CH}_{2}\right)_{3} \mathrm{CH}_{3}, \mathbf{X X I} \\
& \mathrm{R}=-\left(\mathrm{CH}_{2}\right)_{5} \mathrm{CH}_{3}, X X \mathrm{XI}
\end{aligned}
$$

Figure 4. Some examples of novel platinum(II) and platinum(IV) complexes evaluated for their cytotoxic activity on MPM.

\section{MPM immunotherapy}

Pembrolizumab is a high affinity, humanized monoclonal antibody against PD-1 designed to block the interaction between PD-1 and both PD-L1 and PD-L2. ${ }^{48,49}$ It is currently approved for advanced tumors, including advanced non-small-cell lung cancer (NSCLC) with a PD-L1 tumor proportion score of 50\% or more, no EGFR or ALK 
genomic aberrations, and no previous systemic therapy as well as advanced NSCLC with a PD-L1 tumor proportion score of $1 \%$ or more progressing on or after platinum containing chemotherapy. ${ }^{48,49}$

PD-L1 is expressed in up to $40 \%$ of patients suffering from malignant pleural mesothelioma and seems to be more common in non-epithelioid tumors. ${ }^{49}$ PD-L1positive malignant pleural mesothelioma appears to be associated with worse prognosis when compared to PD-L1-negative disease and is an independent risk factor for overall survival. ${ }^{50}$

Initial results from non-randomized, open-label, phase $1 \mathrm{~b}$ trial show that pembrolizumab might be safe for the treatment of malignant pleural mesothelioma, with encouraging signs of anti-tumor activity in this population. ${ }^{51}$

Nivolumab is a fully human monoclonal antibody that binds PD-1 on activated immune cells and disrupts binding of PD-1 to its ligand PD-L1. In recent a single arm phase II trial nivolumab has been shown to have meaningful clinical activity and an acceptable safety profile in second line unselected population of patients with mesothelioma. Further studies with a combination of checkpoint inhibitors ipilimumab and nivolumab - are ongoing. ${ }^{51}$ 
MPM mesenchymal stromal cell experimental treatment

Recent reports have shown that mesenchymal stromal cells (MSCs) are able to migrate specifically towards solid neoplasms and their metastatic localizations when injected intravenously. This peculiar cancer tropism has opened up an emerging field to use MSCs as vectors to deliver antineoplastic drugs for targeted therapies. ${ }^{8}$ 
Mesenchymal stromal cells (MSCs) are a group of undifferentiated multipotent adult cells residing within the human body; they are commonly described as plasticadherent, fibroblast-like cells disclosing clear self-renewal properties and the possibility to differentiate both in vivo and in vitro into a variety of mesenchymal lineage cells; they can differentiate into chondrogenic, osteogenic and adipogenic lineages when adequately cultured in selective media under specific inducing conditions. ${ }^{52,53}$

Once inserted in the host tissues, MSCs can interact with the surrounding microenvironment stimulating tissue healing, reparation and regeneration and exerting intense anti-inflammatory and immunomodulatory effects by several different mechanisms. At the beginning MSCs were thought to be only in bone marrow, but nowadays we know that MSCs can be isolated and characterized from many other adult and fetal tissues, including adipose tissue, dental pulp, umbilical cord, liver, tendon, thymus, cornea, brain, periosteum, spleen, placenta and synovial and amniotic fluids. Although no significant qualitative difference in the profiles of secreted cytokines by different type of MSCs has been reported, several quantitative differences in the cytokine secretions by adipose tissue-derived MSCs (AT-MSCs) and bone marrow-derived MSC (BM-MSC) have been observed. MSCs are able to migrate and engraft at sites of inflammation, tumors and injury in response to cytokines, chemokines and growth factors at a wound site; they can express local restoration 
properties through trans-differentiation or mainly by the paracrine secretion of soluble factors with anti-inflammatory and wound-healing actions. ${ }^{54,55}$

The scope of chemotherapy is to maximize the drug effect in the neoplasm microenvironment to destroy as many cancer cells as possible with the lowest collateral damage. Nanovector of anticancer compounds have been recently developed, potentially improving the free-form drugs activity for several aspects: in fact, they can hide and protect the drug from being metabolized in the body before reaching their target site, thus enhancing tumor drug uptake and reducing drugs interaction with normal cells thereby decreasing clinical toxicity. ${ }^{56}$ Equivalent results can be reached by using MSCs for anti-tumor agent delivery, even considering that MSCs themselves generate antineoplastic factors able to kill cancer cells both in vitro and in vivo.

We have previously demonstrated that paclitaxel-primed mesenchymal stromal cells and a cationic platinum(II) complex- primed mesenchymal stromal cells successfully inhibit the in vitro proliferation of human mesothelioma cells. ${ }^{8,57}$ 


\section{Other thoracic malignancies}

Although we focused our review on NSCLC and MPM, at least two other thoracic malignancies should also be mentioned to complete the clinical scenario of thoracic oncology: small cell lung cancer and thymic neoplasms.

\section{Small cell lung cancer}

Small cell lung cancer is a malignant primary pulmonary tumor belonging to neuroendocrine tumors, although presenting distinct clinical features, treatments and prognosis. 
Limited disease has been historically treated by concomitant chemoradiotherapy with accelerated hyperfractionated radiation therapy concurrently with platinum-based chemotherapy; in case of extensive disease cisplatin is usually combined with either etoposide or irinotecan. ${ }^{58}$

More recently, the safety and efficacy of pembrolizumab and nivolumab in advanced SCLC with at least 1\% PD-L1 expression have been tested with results suggesting promising activity in patients with previously treated SCLC. ${ }^{59}$

Rovalpituzumab - which is a delta like Notch canonical ligand 3 (DLL3)-targeted antibody drug conjugate - was developed in patients with recurrent SCLC, showing a promising result in patients with high DLL3 expression, representing $67 \%$ of the overall population. ${ }^{59}$

Other agents like Tarextumab (a Notch-inhibitor), Veliparib (a poly(ADP-ribose) polymerase), Trilaciclib (a cyclin-dependent kinase 4 and cyclin-dependent kinase 6 inhibitor) have been tested in SCLC patients but results are too preliminary to draw definitive conclusions. ${ }^{59}$

\section{Thymic neoplasms}


Thymic neoplasms show a wide heterogeneity, being thymoma and thymic carcinoma the most frequent histological subtypes; the possibility to obtain complete resection is the most important aspect of the treatment and it is considered the gold standard. ${ }^{60}$

Platinum-containing chemotherapy has been the standard treatment; more recently durvalumab has been tested as well as pembrolizumab in patients with refractory thymic epithelial tumors with an overall response rate of $24 \% .{ }^{59}$

\section{EXPERT OPINION}

Molecular targeted therapy, immunotherapy and mesenchymal stromal cell drug loading and delivery are the new alternatives to standard chemotherapy; molecular targeted therapy - like EGFR TKIs - or immunotherapy - like pembrolizumab or nivolumab - are already a daily clinical reality while, on the contrary, MSC drug loading and delivery are at a preclinical phase. Anyway, both have shown excellent results that lead us to foresee a reduction of standard chemotherapy and an increase of less toxic and dangerous treatments.

Pembrolizumab - a high affinity, humanized monoclonal antibody against PD-1 designed to block the interaction between PD-1 and both PD-L1 and PD-L2 - and Nivolumab - a fully human monoclonal antibody that binds PD-1 on activated immune cells and disrupts binding of PD-1 to its ligand PD-L1 - represent some of the most innovative and promising compounds in the field of immunotherapy although EGFR TKIs still represent an excellent treatment option in responder patients. 
Atezolizumab, a humanized anti-PD-L1 monoclonal immunoglobulin G1 antibody, is currently used in many kinds of advanced carcinoma including metastatic non-small cell lung cancer; moreover Durvalumab, a human IgG1 anti-PD-1 monoclonal antibody, has been approved for the treatment of patients with locally-advanced, unresectable NSCLC whose disease has not progressed following platinum-based chemoradiation therapy.

In the short-term we expect a boost of immunotherapy compounds for NSCLC and MPM and probably many other neoplasms of different districts will be further treated with these regimens.

With the development of immune checkpoint inhibitors, clinicians will have to rethink their evaluation of response to treatment by objective radiological findings: in fact, the so-called pseudoprogression has appeared as a relatively uncommon but concrete phenomenon, representing an objective tumor growth or appearance of new lesions, followed by tumor shrinkage.

A deeper knowledge of the tumor microenvironment led to the emergence of immune checkpoint inhibitors that negatively regulate immune cells and bring to a dormant state, thus being immune cells unable to interact with their targets.

Although immune checkpoint inhibitors are effective when used alone, several combined therapies exist. A better knowledge of the immune checkpoint biology and 
of immune response against tumors, will lead to design many new compounds and therapeutic approaches in few years.

Mesenchymal stromal cells are a new promising tool in the field of drug loading and delivery in oncology: they can migrate and engraft at sites of inflammation, tumors and injury in response to cytokines, chemokines, and growth factors at a wound site; they can express local restoration properties through trans-differentiation or mainly by the paracrine secretion of soluble factors with anti-inflammatory and woundhealing actions.

Although not yet utilized in the clinical practice, we think they will represent another main tool for cancer therapy and it will probably play a leading role in the field of nanovectors and molecular medicine. 


\section{CONCLUSION}

Non-small cell lung cancer (NSCLC) and malignant pleural mesothelioma (MPM) represent the most intriguing and challenging diseases at the moment for thoracic oncologists and surgeons. On one hand, NSCLC is the leading cause of cancer-related death worldwide, but many new drugs and compounds have been recently developed with very interesting results; on the other hand, MPM - although rare - is a fatal asbestos-related tumor without any approved second-line treatments, thus representing a disease setting to test the efficacy of new therapeutic agents. 


\section{ARTICLE HIGHLIGHTS BOX}

- Lung cancer is the leading cause of cancer-related death worldwide and malignant pleural mesothelioma - although rare - is fatal asbestos-related tumor without any approved second-line treatments. Together they are, for different reasons, the most challenging diseases for thoracic surgeons nowadays.

- Although chemotherapy is still indicated in several non-small cell lung cancer, molecular targeted therapy and immunotherapy represent the new frontiers of daily clinical practice.

- Activating mutations in the epidermal growth factor receptor (EGFR) gene and translocations of the anaplastic lymphoma kinase (ALK) gene are the most important mutations with concrete clinical implications, although many others are known.

- Immunotherapy has been now established as one of the most promising therapeutic options both in NSCL and MPM; immune-checkpoint inhibitors 
have meaningful clinical activity and an acceptable safety profile both in NSCLC and MPM.

- Nanovector of anticancer compounds as well as mesenchymal stromal cells can potentially hide and protect the drug from being metabolized in the body before reaching their target site, thus enhancing tumor drug uptake and reducing drugs interaction with normal cells thereby decreasing clinical toxicity. They can be considered the future of antineoplastic drug development.

\section{FIGURE LEGEND}

Figure 1: example of new compounds under evaluation for the treatment of NSCLC

Figure 2: some examples of novel platinum(II) and platinum(IV) complexes evaluated on NSCLC

Figure 3: example of new compounds under evaluation for the treatment of MPM

Figure 4: some examples of novel platinum(II) and platinum(IV) complexes evaluated for their cytotoxic activity on MPM 


\section{REFERENCES}

1. Gridelli C, Rossi A, Carbone DP, Guarize J, Karachaliou N, Mok T, et al. Non-small-cell lung cancer. Nature Reviews Disease Primers 2015 05/21/online;1:15009.

2. Govindan R, Page N, Morgensztern D, Read W, Tierney R, Vlahiotis A, et al. Changing Epidemiology of Small-Cell Lung Cancer in the United States Over the Last 30 Years: Analysis of the Surveillance, Epidemiologic, and End Results Database. Journal of Clinical Oncology 2006;24(28):4539-44. 3. Colby TV, Wistuba II, Gazdar A. Precursors to Pulmonary Neoplasia. Advances in Anatomic Pathology 1998;5(4):205-15.

4. Subramanian J, Govindan R. Lung Cancer in Never Smokers: A Review. Journal of Clinical Oncology 2007;25(5):561-70.

5. Borri A, Leo F, Veronesi G, Solli P, Galetta D, Gasparri R, et al. Extended pneumonectomy for nonsmall cell lung cancer: Morbidity, mortality, and long-term results. The Journal of Thoracic and Cardiovascular Surgery 2007 2007/11/01/;134(5):1266-72.

6. Spaggiari L, Galetta D, Veronesi G, Leo F, Gasparri R, Petrella F, et al. Superior vena cava replacement for lung cancer using a heterologous (bovine) prosthesis: Preliminary results. The Journal of Thoracic and Cardiovascular Surgery 2006 2006/02/01/;131(2):490-91.

7. Petrella F, Chieco P, Solli P, Veronesi G, Borri A, Galetta D, et al. Which factors affect pulmonary function after lung metastasectomy? ? 2 . European Journal of Cardio-Thoracic Surgery 2009;35(5):792-96.

8. Petrella F, Coccè V, Masia C, Milani M, Salè EO, Alessandri G, et al. Paclitaxel-releasing mesenchymal stromal cells inhibit in vitro proliferation of human mesothelioma cells. Biomed Pharmacother 2017 2017/03/01/;87(Supplement C):755-58.

9. Bini A, Grazia M, Petrella F, Chittolini M. Multiple chondromatous hamartomas of the lung. Interactive CardioVascular and Thoracic Surgery 2002;1(2):78-80.

10. Britton M. The epidemiology of mesothelioma. Seminars in Oncology 2002 2002/02/01/;29(1):1825.

11. Novello S, Pinto C, Torri V, Porcu L, Di Maio M, Tiseo M, et al. The Third Italian Consensus Conference for Malignant Pleural Mesothelioma: State of the art and recommendations. Crit Rev Oncol Hematol 2016 2016/08/01/;104:9-20.

12. Azzoli CG, Temin S, Aliff T, Jr SB, Brahmer J, Johnson DH, et al. 2011 Focused Update of 2009 American Society of Clinical Oncology Clinical Practice Guideline Update on Chemotherapy for Stage IV Non-Small-Cell Lung Cancer. Journal of Clinical Oncology 2011;29(28):3825-31.

13. Scagliotti GV, Parikh P, Pawel Jv, Biesma B, Vansteenkiste J, Manegold C, et al. Phase III Study Comparing Cisplatin Plus Gemcitabine With Cisplatin Plus Pemetrexed in Chemotherapy-Naive Patients With Advanced-Stage Non-Small-Cell Lung Cancer. Journal of Clinical Oncology 2008;26(21):3543-51.

14. Sandler A, Gray R, Perry MC, Brahmer J, Schiller JH, Dowlati A, et al. Paclitaxel-Carboplatin Alone or with Bevacizumab for Non-Small-Cell Lung Cancer. New England Journal of Medicine 2006;355(24):254250. 
15. Paz-Ares LG, Marinis Fd, Dediu M, Thomas M, Pujol J-L, Bidoli P, et al. PARAMOUNT: Final Overall Survival Results of the Phase III Study of Maintenance Pemetrexed Versus Placebo Immediately After Induction Treatment With Pemetrexed Plus Cisplatin for Advanced Nonsquamous Non-Small-Cell Lung Cancer. Journal of Clinical Oncology 2013;31(23):2895-902.

16. Kang $F, A i Y$, Zhang $Y$, Huang Z. Design and synthesis of new hybrids from 2-cyano-3,12dioxooleana- 9-dien-28-oic acid and O2-(2,4-dinitrophenyl) diazeniumdiolate for intervention of drugresistant lung cancer. Eur J Med Chem 2018 2018/04/10/;149:269-80.

17. Li X, Guise CP, Taghipouran R, Yosaatmadja Y, Ashoorzadeh A, Paik W-K, et al. 2-Oxo-3, 4dihydropyrimido[4, 5-d]pyrimidinyl derivatives as new irreversible pan fibroblast growth factor receptor (FGFR) inhibitors. Eur J Med Chem 2017 2017/07/28/;135:531-43.

18. Chamni S, Sirimangkalakitti N, Chanvorachote P, Saito N, Suwanborirux K. Chemistry of Renieramycins. 17. A New Generation of Renieramycins: Hydroquinone 5-O-Monoester Analogues of Renieramycin M as Potential Cytotoxic Agents against Non-Small-Cell Lung Cancer Cells. Journal of Natural Products 2017 2017/05/26;80(5):1541-47.

19. Yin J-Y, Li X, Zhou H-H, Liu Z-Q. Pharmacogenomics of platinum-based chemotherapy sensitivity in NSCLC: toward precision medicine. Pharmacogenomics 2016;17(12):1365-78.

20. Rahman F-U, Bhatti MZ, Ali A, Duong H-Q, Zhang Y, Yang B, et al. Homo- and heteroleptic Pt(II) complexes of ONN donor hydrazone and 4-picoline: A synthetic, structural and detailed mechanistic anticancer investigation. Eur J Med Chem 2018 2018/01/01/;143:1039-52.

21. Sieste S, Lifincev I, Stein N, Wagner G. Synthesis, characterisation and in vitro cytotoxicity of mixed ligand Pt(ii) oxadiazoline complexes with hexamethylenetetramine and 7-nitro-1,3,5-triazaadamantane. Dalton Trans 2017;46(36):12226-38.

22. Huang X, Huang R, Gou S, Wang Z, Liao Z, Wang H. Platinum(IV) complexes conjugated with phenstatin analogue as inhibitors of microtubule polymerization and reverser of multidrug resistance. Bioorg Med Chem 2017 2017/09/01/;25(17):4686-700.

23. Bauer E, Domingo X, Balcells C, Polat IH, Crespo M, Quirante J, et al. Synthesis, characterization and biological activity of new cyclometallated platinum(iv) iodido complexes. Dalton Trans 2017;46(43):1497387.

24. Pao W, Miller VA. Epidermal Growth Factor Receptor Mutations, Small-Molecule Kinase Inhibitors, and Non-Small-Cell Lung Cancer: Current Knowledge and Future Directions. Journal of Clinical Oncology 2005;23(11):2556-68.

25. Mok T, Yang J-J, Lam K-C. Treating Patients With EGFR-Sensitizing Mutations: First Line or Second Line-Is There a Difference? Journal of Clinical Oncology 2013;31(8):1081-88.

26. Chong CR, Jänne PA. The quest to overcome resistance to EGFR-targeted therapies in cancer. Nature Medicine 2013 11/07/online;19:1389.

27. Soda M, Choi YL, Enomoto M, Takada S, Yamashita Y, Ishikawa S, et al. Identification of the transforming EML4-ALK fusion gene in non-small-cell lung cancer. Nature 2007 07/11/online;448:561.

28. Shaw AT, Ou S-HI, Bang Y-J, Camidge DR, Solomon BJ, Salgia R, et al. Crizotinib in ROS1-Rearranged Non-Small-Cell Lung Cancer. New England Journal of Medicine 2014;371(21):1963-71.

29. Rybarczyk-Kasiuchnicz A, Ramlau R. Current views on molecularly targeted therapy for lung cancer - a review of literature from the last five years. Kardiochirurgia i Torakochirurgia Polska/Polish Journal of Thoracic and Cardiovascular Surgery 2018;15(2):119-24.

30. Nguyen-Ngoc T, Bouchaab H, Adjei AA, Peters S. BRAF Alterations as Therapeutic Targets in NonSmall-Cell Lung Cancer. Journal of Thoracic Oncology 2015 2015/10/01/;10(10):1396-403.

31. Hayashi $\mathrm{H}$, Nakagawa K. Current evidence in support of the second-generation anaplastic lymphoma kinase ( ALK) tyrosine kinase inhibitor alectinib for the treatment of non-small cell lung cancer positive for ALK translocation. Journal of Thoracic Disease 2016;8(10):E1311-E16.

32. Raju S JR, Sehgal S Review of checkpoint immunotherapy for the management of non-small cell lung cancer. ImmunoTargets and Therapy 2018;7 63-75.

33. Pardoll DM. The blockade of immune checkpoints in cancer immunotherapy. Nature Reviews Cancer 2012 03/22/online;12:252. 
34. D HR. Immunologic and Clinical Effects of Targeting PD-1 in Lung Cancer. Clinical Pharmacology \& Therapeutics 2014;96(2):214-23.

35. Hellmann MD, Ciuleanu T-E, Pluzanski A, Lee JS, Otterson GA, Audigier-Valette C, et al. Nivolumab plus Ipilimumab in Lung Cancer with a High Tumor Mutational Burden. New England Journal of Medicine 2018;378(22):2093-104.

36. Vogelzang NJ, Rusthoven JJ, Symanowski J, Denham C, Kaukel E, Ruffie P, et al. Phase III Study of Pemetrexed in Combination With Cisplatin Versus Cisplatin Alone in Patients With Malignant Pleural Mesothelioma. Journal of Clinical Oncology 2003;21(14):2636-44.

37. Facchetti G, Petrella F, Spaggiari L, Rimoldi I. Malignant Pleural Mesothelioma: State of the art and advanced cell therapy. Eur J Med Chem 2017 2017/12/15/;142(Supplement C):266-70.

38. Alley EW, Lopez J, Santoro A, Morosky A, Saraf S, Piperdi B, et al. Clinical safety and activity of pembrolizumab in patients with malignant pleural mesothelioma (KEYNOTE-028): preliminary results from a non-randomised, open-label, phase $1 \mathrm{~b}$ trial. Lancet Oncol 2017 2017/05/01/;18(5):623-30.

39. Ceresoli GL, Zucali PA, De Vincenzo F, Gianoncelli L, Simonelli M, Lorenzi E, et al. Retreatment with pemetrexed-based chemotherapy in patients with malignant pleural mesothelioma. Lung Cancer 2011 2011/04/01/;72(1):73-77.

40. Marcella B, Daniele C, Riccardo I, Paola I, Asadoor N, Maria BF, et al. Possible repurposing of pyrvinium pamoate for the treatment of mesothelioma: A pre-clinical assessment. Journal of Cellular Physiology 2018;233(9):7391-401.

41. Yoshiko K, Hisao N, Ayako T, Takeshi K, Akinobu G, Akito T, et al. Newly synthesized anticancer drug HUHS1015 is effective on malignant pleural mesothelioma. Cancer Science 2014;105(7):883-89.

42. Cincinelli R, Musso L, Artali R, Guglielmi M, Bianchino E, Cardile F, et al. Camptothecin-psammaplin A hybrids as topoisomerase I and HDAC dual-action inhibitors. Eur J Med Chem 2018 2018/01/01/;143:2005-14.

43. Ang WH, Khalaila I, Allardyce CS, Juillerat-Jeanneret L, Dyson PJ. Rational Design of Platinum(IV) Compounds to Overcome Glutathione-S-Transferase Mediated Drug Resistance. J Am Chem Soc 2005 2005/02/01;127(5):1382-83.

44. Zanellato I, Bonarrigo I, Sardi M, Alessio M, Gabano E, Ravera M, et al. Evaluation of PlatinumEthacrynic Acid Conjugates in the Treatment of Mesothelioma. ChemMedChem 2011;6(12):2287-93.

45. Gabano E, Ravera M, Trivero F, Tinello S, Gallina A, Zanellato I, et al. The cisplatin-based Pt(iv)diclorofibrato multi-action anticancer prodrug exhibits excellent performances also under hypoxic conditions. Dalton Trans 2018;47(25):8268-82.

46. Ravera M, Gabano E, Zanellato I, Bonarrigo I, Escribano E, Moreno V, et al. Synthesis, characterization and antiproliferative activity on mesothelioma cell lines of bis(carboxylato)platinum(iv) complexes based on picoplatin. Dalton Trans 2012;41(11):3313-20.

47. Rimoldi I, Facchetti G, Lucchini G, Castiglioni E, Marchianò S, Ferri N. In vitro anticancer activity evaluation of new cationic platinum(II) complexes based on imidazole moiety. Bioorg Med Chem 2017;25(6):1907-13.

48. Sul J, Blumenthal GM, Jiang X, He K, Keegan P, Pazdur R. FDA Approval Summary: Pembrolizumab for the Treatment of Patients With Metastatic Non-Small Cell Lung Cancer Whose Tumors Express Programmed Death-Ligand 1. The Oncologist 2016 May 1, 2016;21(5):643-50.

49. Garon EB, Rizvi NA, Hui R, Leighl N, Balmanoukian AS, Eder JP, et al. Pembrolizumab for the Treatment of Non-Small-Cell Lung Cancer. New England Journal of Medicine 2015;372(21):2018-28.

50. Mansfield AS, Roden AC, Peikert T, Sheinin YM, Harrington SM, Krco CJ, et al. B7-H1 Expression in Malignant Pleural Mesothelioma is Associated with Sarcomatoid Histology and Poor Prognosis. Journal of Thoracic Oncology 2014 2014/07/01/;9(7):1036-40.

51. Quispel-Janssen J, van der Noort V, de Vries JF, Zimmerman M, Lalezari F, Thunnissen E, et al. PD-1 blockade with nivolumab in patients with recurrent Malignant Pleural Mesothelioma. Journal of Thoracic Oncology 2018 2018/06/14/.

52. Petrella F, Rizzo S, Borri A, Casiraghi M, Spaggiari L. Current Perspectives in Mesenchymal Stromal Cell Therapies for Airway Tissue Defects. Stem Cells Int 2015;2015:7. 
53. Rizzo S, Petrella F, Zucca I, Rinaldi E, Barbaglia A, Padelli F, et al. In vitro labelling and detection of mesenchymal stromal cells: a comparison between magnetic resonance imaging of iron-labelled cells and magnetic resonance spectroscopy of fluorine-labelled cells. European Radiology Experimental 2017 June 29;1(1):6.

54. Petrella F, Toffalorio F, Brizzola S, De Pas TM, Rizzo S, Barberis M, et al. Stem Cell Transplantation Effectively Occludes Bronchopleural Fistula in an Animal Model. The Annals of Thoracic Surgery 2013;97(2):480-83.

55. Pessina A, Bonomi A, Coccè V, Invernici G, Navone S, Cavicchini L, et al. Mesenchymal Stromal Cells Primed with Paclitaxel Provide a New Approach for Cancer Therapy. PLOS ONE 2011;6(12):e28321.

56. Hsueh CT SJ, Tsai JY, Hsueh CT. . Nanovectors for anti-cancer drug delivery in the treatment of advanced pancreatic adenocarcinoma. . World J Gastroenterol 2016;22(31):7080-90.

57. Rimoldi I, Coccè V, Facchetti G, Alessandri G, Brini AT, Sisto F, et al. Uptake-release by MSCs of a cationic platinum(II) complex active in vitro on human malignant cancer cell lines. Biomed Pharmacother 2018 2018/12/01/;108:111-18.

58. Spaggiari L PF. Small Cell Lung Cancer ESTS Textbook of Thoracic Surgery: Medycyna Praktyczna 2014:825-32.

59. Ferrara R, Mezquita L, Besse B. Progress in the Management of Advanced Thoracic Malignancies in 2017. Journal of Thoracic Oncology 2018 2018/03/01/;13(3):301-22.

60. Venuta F AM, Vitolo D, Rendina EA, Diso D Thymoma and Thymic Carcinoma Chapter ESTS Textbook of Thoracic Surgery Medycyna Praktyczna 2014:277 - 94. 